J. Indones. Math. Soc.

Vol. 18, No. 1 (2012), pp. 57-66.

\title{
ON THE WIENER INDEX OF A GRAPH
}

\author{
Harishchandra S. Ramane ${ }^{1}$, Deepak S. Revankar ${ }^{2}$, and Asha B. \\ GANAGI $^{3}$ \\ ${ }^{1}$ Department of Mathematics, Gogte Institute of Technology, \\ Udyambag, Belgaum - 590008, India, hsramane@yahoo.com \\ ${ }^{2}$ Department of Mathematics, Gogte Institute of Technology, \\ Udyambag, Belgaum - 590008, India, revankards@rediffmail.com \\ ${ }^{3}$ Department of Mathematics, Gogte Institute of Technology, \\ Udyambag, Belgaum - 590008, India, abganagi@yahoo.co.in
}

\begin{abstract}
The Wiener index of a graph $G$, denoted by $W(G)$ is the sum of the distances between all (unordered) pairs of vertices of $G$. In this paper, we obtain the Wiener index of line graphs and some class of graphs.

Key words: Wiener index, line graph, distance, diameter.
\end{abstract}

\begin{abstract}
Abstrak. Indeks Weiner dari suatu graf $G$, yang dinotasikan dengan $W(G)$ adalah jumlahan jarak antara semua pasangan (tak terurut) dari titik-titik $G$. Pada artikel ini, kami mendapatkan indeks Weiner dari graf garis dan beberapa kelas dari graf.

Kata kunci: Indeks Wiener, graf garis, jarak, diameter.
\end{abstract}

\section{Introduction}

Let $G$ be a simple, connected, undirected graph with vertex set $V(G)=$ $\left\{v_{1}, v_{2}, \ldots, v_{n}\right\}$ and edge set $E(G)=\left\{e_{1}, e_{2}, \ldots, e_{m}\right\}$. The distance between two vertices $v_{i}$ and $v_{j}$, denoted by $d\left(v_{i}, v_{j}\right)$ is the length of shortest path between the vertices $v_{i}$ and $v_{j}$ in $G$. The shortest $v_{i}-v_{j}$ path is often called a geodesic. The diameter $\operatorname{diam}(G)$ of a connected graph $G$ is the length of any longest geodesic. The degree of a vertex $v_{i}$ in $G$ is the number of edges incident to $v_{i}$ and is denoted by $d_{i}=\operatorname{deg}\left(v_{i}\right)[2,11]$.

The Wiener index (or Wiener number) [18] of a graph $G$, denoted by $W(G)$ is the sum of the distances between all (unordered) pairs of vertices of $G$, that is

2000 Mathematics Subject Classification: 05C12.

Received: 07-11-2011, revised: 04-05-2012, accepted: 14-05-2012. 


$$
W(G)=\sum_{i<j} d\left(v_{i}, v_{j}\right) .
$$

The Wiener index is a graph invariant that belongs to the molecules structuredescriptors called topological indices, which are used for the design of molecules with desired properties $[16]$.

If $\mu_{1} \geq \mu_{2} \geq \ldots \geq \mu_{n}$ be the eigenvalues of Laplacian Matrix [13] of a tree $T$, then $[10,12]$

$$
W(T)=n \sum_{i=1}^{n-1} \frac{1}{\mu_{i}}
$$

For details on Wiener index, see $[4,10,14]$.

The line graph $L(G)$ of a graph $G$ is a graph such that the vertices of $L(G)$ are the edges of $G$ and two vertices of $L(G)$ are adjacent if and only if their corresponding edges in $G$ share a common vertex [11]. The concept of line graph has various applications in physical chemistry $[7,9]$.

Let $F_{1}$ be the 5 -vertex path, $F_{2}$ the graph obtained by identifying a vertex of a triangle with an end vertex of the 3 -vertex path, and $F_{3}$ the graph obtained by identifying a vertex of a triangle with a vertex of another triangle (see Fig. 1).

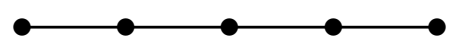

$F_{1}$

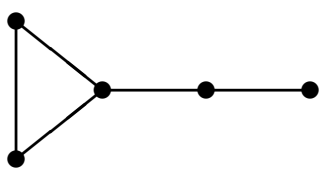

$F_{2}$

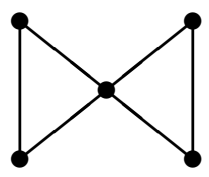

$F_{3}$

Figure 1

Theorem 1.1. [15] If $\operatorname{diam}(G) \leq 2$ and none of the graphs $F_{1}, F_{2}, F_{3}$ of Fig. 1 is an induced subgraph of $G$ then $\operatorname{diam}(L(G)) \leq 2$.

Recently there has been an interest in understanding the connection between $W(G)$ and $W(L(G))$.

Theorem 1.2. [1] For every tree $T$ on $n$ vertices $W(L(T))=W(T)-\left(\begin{array}{c}n \\ 2\end{array}\right)$. 
Theorem 1.3. [6] If $G$ is connected graph with $n$ vertices and $m$ edges then

$$
W(L(G)) \geq W(G)-n(n-1)+\frac{m(m+1)}{2} .
$$

Theorem 1.4. [8] If $G$ is connected unicyclic graph with $n$ vertices then $W(L(G)) \leq$ $W(G)$ with equality if and only if $G$ is a cycle of length $n$.

Theorem 1.5. [3] Let $G$ be a connected graph with minimum degree $\delta(G) \geq 2$ then $W(G) \leq W(L(G))$. Equality holds only for cycles.

Graphs for which $W(G)=W(L(G))$ are considered in [3, 5].

In the sequel, in this paper we obtain some more results on the Weiner index of line graphs. Also, we obtain Wiener index of some class of graphs.

\section{Wiener Index of Line Graphs}

Theorem 2.1. Let $G$ be a connected graph with $n$ vertices, $m$ edges and $d_{i}=$ $\operatorname{deg}\left(v_{i}\right)$. If $\operatorname{diam}(G) \leq 2$ and $G$ does not contain $F_{i}, i=1,2,3$ (of Fig. 1) as an induced subgraph then

$$
W(L(G))=m^{2}-\frac{1}{2} \sum_{i=1}^{n} d_{i}^{2} .
$$

ProOF. The number of vertices of $L(G)$ is $n_{1}=m$ and the number of edges of $L(G)$ is $m_{1}=-m+\frac{1}{2} \sum_{i=1}^{n} d_{i}^{2}[11]$.

If $\operatorname{diam}(G) \leq 2$, then $[17]$

$$
W(G)=n(n-1)-m
$$

From Theorem 1.1, since $\operatorname{diam}(G) \leq 2$ and $G$ has no $F_{i}, i=1,2,3$ as its induced subgraph then $\operatorname{diam}(L(G)) \leq 2$. Therefore from Eq. (1),

$$
\begin{aligned}
W(L(G)) & =n_{1}\left(n_{1}-1\right)-m_{1} \\
& =m(m-1)-\left[-m+\frac{1}{2} \sum_{i=1}^{n} d_{i}^{2}\right] \\
& =m^{2}-\frac{1}{2} \sum_{i=1}^{n} d_{i}^{2} .
\end{aligned}
$$


Corollary 2.2. If $G$ is a connected $r$-regular graph on $n$ vertices with $\operatorname{diam}(G) \leq 2$ and none of $F_{i}, i=1,2,3$ (of Fig. 1) as an induced subgraph of $G$ then,

$$
W(L(G))=\frac{n r^{2}(n-2)}{4}
$$

Proof. Since $G$ is an $r$-regular graph on $n$ vertices, the number of edges of $G$ is $m=n r / 2$ and $d_{i}=\operatorname{deg}\left(v_{i}\right)=r$. From Theorem 2.1,

$$
\begin{aligned}
W(L(G)) & =m^{2}-\frac{1}{2} \sum_{i=1}^{n} d_{i}^{2} \\
& =\left(\frac{n r}{2}\right)^{2}-\frac{1}{2} \sum_{i=1}^{n} r^{2} \\
& =\frac{n^{2} r^{2}}{4}-\frac{n r^{2}}{2}=\frac{n r^{2}(n-2)}{4}
\end{aligned}
$$

Let $e=(u v)$ be an edge of a graph $G$ where $u$ and $v$ are the end vertices of $e$. The degree of edge $e$ is defined as $\operatorname{deg}(e)=\operatorname{deg}(u)+\operatorname{deg}(v)-2$.

Theorem 2.3. Let $G$ be a connected graph with vertex set $V(G)=\left\{v_{1}, v_{2}, \ldots, v_{n}\right\}$ and edge set $E(G)=\left\{e_{1}, e_{2}, \ldots, e_{m}\right\}$. Let $d_{i}=\operatorname{deg}\left(v_{i}\right)$. Then

$$
W(L(G)) \geq \sum_{i=1}^{n} \frac{d_{i}\left(d_{i}-1\right)}{2}+m(m-1)-\sum_{i=1}^{m} \operatorname{deg}\left(e_{i}\right)
$$

The equality holds if and only if $\operatorname{diam}(G) \leq 2$ and none of the three graphs of Fig. 1 is an induced subgraph of $G$.

Proof. If $d_{i}=\operatorname{deg}\left(v_{i}\right)$ then for each vertex $v_{i}$ there are $d_{i}$ edges incident to $v_{i}$. These $d_{i}$ edges form a complete graph on $d_{i}$ vertices in $L(G)$. Which contributes $d_{i}\left(d_{i}-1\right) / 2$ to the $W(L(G))$.

Consider an edge $e=(u v)$ which is adjacent to $\operatorname{deg}(u)+\operatorname{deg}(v)-2=\operatorname{deg}(e)$ edges at $u$ and $v$ taken together. Hence the edge $e$ is not adjacent to remaining $m-1-\operatorname{deg}(e)$ edges of $G$. In $L(G)$ the distance between $e$ and the remaining these $m-1-\operatorname{deg}(e)$ vertices is more than 1 . Hence each edge $e=(u v)$ contributes the distance at least $2(m-1-\operatorname{deg}(e))$ in $L(G)$. Therefore 


$$
\begin{aligned}
W(L(G)) & \geq \sum_{i=1}^{n} \frac{d_{i}\left(d_{i}-1\right)}{2}+\frac{1}{2} \sum_{e \in E(G)} 2(m-1-\operatorname{deg}(e)) \\
& =\sum_{i=1}^{n} \frac{d_{i}\left(d_{i}-1\right)}{2}+\sum_{i=1}^{m}\left(m-1-\operatorname{deg}\left(e_{i}\right)\right) \\
& =\sum_{i=1}^{n} \frac{d_{i}\left(d_{i}-1\right)}{2}+m(m-1)-\sum_{i=1}^{m} \operatorname{deg}\left(e_{i}\right) .
\end{aligned}
$$

For the equality:

If $\operatorname{diam}(G) \leq 2$ and none of the three graphs of Fig. 1 is an induced subgraph of $G$, then from Theorem 1.1, $\operatorname{diam}(L(G)) \leq 2$. Therefore as explained above, the distance between $e$ and the remaining $m-1-\operatorname{deg}(e)$ vertices in $L(G)$ is 2 . Therefore

$$
\begin{aligned}
W(L(G)) & =\sum_{i=1}^{n} \frac{d_{i}\left(d_{i}-1\right)}{2}+\sum_{e \in E(G)}(m-1-\operatorname{deg}(e)) \\
& =\sum_{i=1}^{n} \frac{d_{i}\left(d_{i}-1\right)}{2}+m(m-1)-\sum_{i=1}^{m} \operatorname{deg}\left(e_{i}\right)
\end{aligned}
$$

Conversely, the first part of Eq. (2) contributes the distance betwen the adjacent edges and the second part contributes the distance 2 between non adjacent edges. For this let $e_{i}$ and $e_{j}$ be nonadjacent edges in $G$. Since $d\left(e_{i}, e_{j}\right)=2$ in $L(G)$, there is an edge $e_{k}$ adjacent to $e_{i}$ and $e_{j}$ in $G$ and none of the three graphs of Fig. 1 is an induced subgraph of $G, \operatorname{diam}(G) \leq 2$. Hence $G$ is required graph.

If $G$ is an $r$-regular graph then $d_{i}=r, \operatorname{deg}(e)=2 r-2$ and $m=n r / 2$, so we have following corollary.

Corollary 2.4. If $G$ is a connected $r$-regular graph on $n$ vertices then $W(L G)) \geq$ $n r^{2}(n-2) / 4$ with equality if and only if $G$ is an $r$-regualr graph with $\operatorname{diam}(G) \leq 2$ and none of the three graphs of Fig. 1 is an induced subgraph of $G$.

Theorem 2.5. If $T$ is a tree with vertices $v_{1}, v_{2}, \ldots, v_{n}$ and $d_{i}=\operatorname{deg}\left(v_{i}\right), i=$ $1,2, \ldots, n$ then

$$
W(L(T))=\sum_{i=1}^{n} \frac{d_{i}\left(d_{i}-1\right)}{2}+\sum_{i<j}\left[1+d\left(v_{i}, v_{j}\right)\right]\left(d_{i}-1\right)\left(d_{j}-1\right) .
$$

Proof. Edges of $T$ will be the vertices of $L(T)$. For each vertex $v_{i}$ there are $d_{i}$ edges incident to it. These edges form a complete graph on $d_{i}$ vertices in $L(T)$. Therfore the sum of the distances between these $d_{i}$ vertices is 


$$
\left(\begin{array}{c}
d_{i} \\
2
\end{array}\right)=\frac{d_{i}\left(d_{i}-1\right)}{2}, i=1,2, \ldots, n
$$

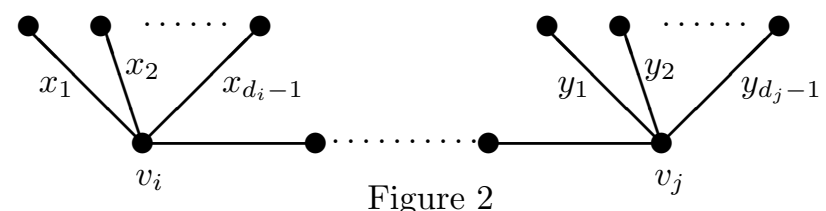

Figure 2

Now suppose $v_{i}$ and $v_{j}$ be the vertices of $T$ and $d_{i}=\operatorname{deg}\left(v_{i}\right)$ and $d_{j}=\operatorname{deg}\left(v_{j}\right)$. Let $x_{1}, x_{2}, \ldots, x_{d_{i}-1}$ be the edges incident to $v_{i}$ and $y_{1}, y_{2}, \ldots, y_{d_{j}-1}$ be the edges incident to $v_{j}$ (Fig. 2). Where $x_{l},\left(1 \leq l \leq d_{i}-1\right)$ and $y_{k},\left(1 \leq k \leq d_{j}-1\right)$ do not have common vertex and these are not the edges of the path between $v_{i}$ and $v_{j}$.

The distance between $x_{l}$ and $y_{k}$ in $L(T)$ is $1+d\left(v_{i}, v_{j}\right)$.

The sum of the distances between all edges $x_{1}, x_{2}, \ldots, x_{d_{i}-1}$ incident to $v_{i}$ and all edges $y_{1}, y_{2}, \ldots, y_{d_{j}-1}$ incidnet to $v_{j}$ is

$$
\left[1+d\left(v_{i}, v_{j}\right)\right]\left(d_{i}-1\right)\left(d_{j}-1\right)
$$

Thus from Eq. (4) and Eq. (5),

$$
W(L(T))=\sum_{i=1}^{n} \frac{d_{i}\left(d_{i}-1\right)}{2}+\sum_{i<j}\left[1+d\left(v_{i}, v_{j}\right)\right]\left(d_{i}-1\right)\left(d_{j}-1\right) .
$$

Theorem 2.6. If $T$ is a tree having $k$ vertices with degree $s$ and remaining with degree 1. Then

$$
W(L(T))=\frac{k s(s-1)}{2}+(s-1)^{2}\left[\left(\begin{array}{l}
k \\
2
\end{array}\right)+W\left(T^{\prime}\right)\right]
$$

where $T^{\prime}$ is the tree obtained from $T$ by removing all its end vertices.

PROOF. The $k$ vertices are of degree $s$ and the remaining $n-k$ vertices are of degree 1. Say $\operatorname{deg}\left(v_{i}\right)=s$ for $i=1,2, \ldots, k$ and $\operatorname{deg}\left(v_{i}\right)=1$ for $i=k+1, k+2, \ldots, n$. So $d_{i}-1=0$ and $d_{j}-1=0$ for $i, j=k+1, k+2, \ldots, n$. From Eq. (3), 


$$
\begin{aligned}
W(L(T)) & =\sum_{i=1}^{k} \frac{s(s-1)}{2}+\sum_{1 \leq i<j \leq k}\left[1+d\left(v_{i}, v_{j}\right)\right](s-1)(s-1) \\
& =\frac{k s(s-1)}{2}+\sum_{1 \leq i<j \leq k}(s-1)^{2}+\sum_{1 \leq i<j \leq k}(s-1)^{2} d\left(v_{i}, v_{j}\right) \\
& =\frac{k s(s-1)}{2}+(s-1)^{2}[(k-1)+(k-2)+\ldots+1]+(s-1)^{2} \sum_{1 \leq i<j \leq k} d\left(v_{i}, v_{j}\right) \\
& =\frac{k s(s-1)}{2}+(s-1)^{2} \frac{(k-1) k}{2}+(s-1)^{2} W\left(T^{\prime}\right) \\
& \left.=\frac{k s(s-1)}{2}+(s-1)^{2}\left[\left(\begin{array}{c}
k \\
2
\end{array}\right)+W\left(T^{\prime}\right)\right)\right]
\end{aligned}
$$

\section{Wiener Index of Some Class of Graphs}

The clique of a graph $G$ is the maximal complete induced subgraph of $G$ [11].

Theorem 3.1. Let $G$ be a connected graph with $n$ vertices having a clique $K_{k}$ of order $k$. Let $G(n, k)$ be the graph obtained from $G$ by removing the edges of $K_{k}$, $0 \leq k \leq n-1$. Then

$$
W(G(n, k)) \geq \frac{1}{2}[n(n-1)+k(k-1)] .
$$

The equality holds if and only if $G \cong K_{n}$, a complete graph on $n$ vertices.

Proof. Let the vertices of $G$ be $v_{1}, v_{2}, \ldots, v_{n}$. Without loss of generality, let the vertex set of the clique $K_{k}$ of $G$ be $S_{1}=\left\{v_{1}, v_{2}, \ldots, v_{k}\right\}$ and the remaining vertices of $G$ are $v_{k+1}, v_{k+2}, \ldots, v_{n}$.

$$
\text { In } G(n, k), d\left(v_{i}, v_{j}\right) \geq 2 \text {, if } v_{i}, v_{j} \in S_{1} \text { and } d\left(v_{i}, v_{j}\right) \geq 1 \text {, otherwise. So }\left(\begin{array}{c}
k \\
2
\end{array}\right)
$$

pairs of vertices are at distance greater than or equal to 2 and remaining $\left(\begin{array}{c}n \\ 2\end{array}\right)$ $\left(\begin{array}{c}k \\ 2\end{array}\right)$ pairs of vertices are at distance greater than or equal to 1 . Therefore

$$
\begin{aligned}
W(G(n, k)) & =\sum_{i<j} d\left(v_{i}, v_{j}\right) \\
& \geq(2)\left(\begin{array}{c}
k \\
2
\end{array}\right)+(1)\left[\left(\begin{array}{c}
n \\
2
\end{array}\right)-\left(\begin{array}{c}
k \\
2
\end{array}\right)\right] \\
& =\frac{1}{2}[n(n-1)+k(k-1)] .
\end{aligned}
$$


For the equality, if $G=K_{n}$, then in $G(n, k), d\left(v_{i}, v_{j}\right)=2$ if $v_{i}, v_{j} \in S_{1}$ and $d\left(v_{i}, v_{j}\right)=1$, otherwise. So

$$
\begin{aligned}
W(G(n, k)) & =(2)\left(\begin{array}{c}
k \\
2
\end{array}\right)+(1)\left[\left(\begin{array}{c}
n \\
2
\end{array}\right)-\left(\begin{array}{c}
k \\
2
\end{array}\right)\right] \\
& =\frac{1}{2}[n(n-1)+k(k-1)] .
\end{aligned}
$$

Conversely, let $W(G(n, k))=\frac{1}{2}[n(n-1)+k(k-1)]$.

Let $G \neq K_{n}$, then there exists at least one pair of vertices which are not adjacent. Let $v_{1}, v_{2}, \ldots, v_{k}$ be the vertices of the clique $K_{k}$ of $G$. Let $v_{k+1}, v_{k+2}, \ldots, v_{k+l}$ be the vertices which are not adjacent among themselves in $G$, where $2 \leq l \leq n-k$.

Let $V_{1}=\left\{v_{1}, v_{2}, \ldots, v_{k}\right\}, V_{2}=\left\{v_{k+1}, v_{k+2}, \ldots, v_{k+l}\right\}$ and $V_{3}=\left\{v_{k+l+1}, v_{k+l+2}, \ldots, v_{n}\right\}$.

In $G(n, k), d\left(v_{i}, v_{j}\right) \geq 2$ if $v_{i}, v_{j} \in V_{1}, d\left(v_{i}, v_{j}\right) \geq 2$ if $v_{i}, v_{j} \in V_{2}$ and $d\left(v_{i}, v_{j}\right) \geq 1$, otherwise. Therefore

$$
\begin{aligned}
W(G(n, k)) & \geq(2)\left(\begin{array}{c}
k \\
2
\end{array}\right)+(2)\left(\begin{array}{l}
l \\
2
\end{array}\right)+(1)\left[\left(\begin{array}{l}
n \\
2
\end{array}\right)-\left(\begin{array}{l}
k \\
2
\end{array}\right)-\left(\begin{array}{l}
l \\
2
\end{array}\right)\right] \\
& =\frac{1}{2}[n(n-1)+k(k-1)+l(l-1)] \\
& \geq \frac{1}{2}[n(n-1)+k(k-1)+2(2-1)] \quad \text { since } l \geq 2 \\
& \left.=\frac{1}{2}[n(n-1)+k(k-1)+2)\right] .
\end{aligned}
$$
$G=K_{n}$

Which is a contradiction to $W(G(n, k))=\frac{1}{2}[n(n-1)+k(k-1)]$. Hence

Two subgraphs $G_{1}$ and $G_{2}$ of $G$ with the vertex sets $V\left(G_{1}\right)$ and $V\left(G_{2}\right)$ respectively are said to be independent if $V\left(G_{1}\right) \cap V\left(G_{2}\right)=\phi$.

Theorem 3.2. Let $\left(K_{p}\right)_{i}, i=1,2, \ldots, k$ be the $k$ independent complete subgraphs on $p$ vertices of $K_{n}$. Let $G(n, p, k)$ be the graph obtained from complete graph $K_{n}$ by removing the edges of $\left(K_{p}\right)_{i}, i=1,2, \ldots, k, 1 \leq k \leq\lfloor n / p\rfloor$ and $0 \leq p \leq n-1$, then

$$
W\left(G(n, p, k)=\frac{n(n-1)+k p(p-1)}{2} .\right.
$$

Proof. Let $\left(K_{p}\right)_{1},\left(K_{p}\right)_{2}, \ldots,\left(K_{p}\right)_{k}$ be the independent subgraphs of $K_{n}$. Let $v_{(i-1) p+1}, v_{(i-1) p+2}, \ldots, v_{(i-1) p+p}$ be the vertices of $\left(K_{p}\right)_{i}, i=1,2, \ldots, k$. So in $G(n, p, k)$ there are $k p(p-1) / 2$ pairs of vertices are at distance 2 and remaining $\left(\begin{array}{c}n \\ 2\end{array}\right)-\frac{k p(p-1)}{2}$ pairs of vertices are at distance 1 . Therefore 


$$
\begin{aligned}
W(G(n, p, k) & =\sum_{i<j} d\left(v_{i}, v_{j}\right) \\
& =(2) \frac{k p(p-1)}{2}+(1)\left[\left(\begin{array}{c}
n \\
2
\end{array}\right)-\frac{k p(p-1)}{2}\right] \\
& =\frac{n(n-1)+k p(p-1)}{2} .
\end{aligned}
$$

Theorem 3.3. Let $e_{i}, i=1,2, \ldots, k, 0 \leq k \leq n-2$ be the edges of complete graph $K_{n}$ incident to a vertex $v$ of $K_{n}$. Let $K_{n}(k)$ be the graph obtained from $K_{n}$ by removing the edges $e_{i}, i=1,2, \ldots, k$. Then

$$
W\left(K_{n}(k)\right)=\left(\begin{array}{c}
n \\
2
\end{array}\right)+k
$$

Proof. Let $v$ is adjacent to $v_{1}, v_{2}, \ldots, v_{k}$ in the complete graph $K_{n}$. Therefore in $K_{n}(k)$ there are $k$ pairs of vertices which are at distance 2 and remaining $\left(\begin{array}{c}n \\ 2\end{array}\right)-k$ pairs of vertices are at distance 1 . Therefore

$$
\begin{aligned}
W\left(K_{n}(k)\right) & =2 k+\left[\left(\begin{array}{c}
n \\
2
\end{array}\right)-k\right] \\
& =\left(\begin{array}{c}
n \\
2
\end{array}\right)+k .
\end{aligned}
$$

Acknowledgement The author HSR is thankful to Gogte Institute of Technology, Belgaum, India and Visvesvaraya Technological University, Belgaum, India, for their support through financial assistance and support through Research Grants Scheme (No. VTU/Aca-RGS/2008-2009/7198).

\section{References}

[1] Buckley, F., "Mean distance of line graphs", Congr. Numer. 32 (1981), 153 - 162.

[2] Buckley, F. and Harary, F., Distance in Graphs, Addison - Wesley, Redwood, 1990.

[3] Cohen, N., Dimitrove, D., Krakovski, R., Škrekovski, R. and Vulkašinović, V., "On Wiener index of graphs and their line graphs", Inst. Math. Phys. Mech. 48 (2010), 1113.

[4] Dobrynin, A.A., Entringer, R. and Gutman, I., "Wiener index of trees: theory and applications", Acta Appl. Math. 66 (2001), 211 - 249.

[5] Dobrynin, A.A. and Melnikov, L.S., "Some results on the Wiener index of iterated line graphs", Electron. Notes Discrete Math. 22 (2005), 469 - 475.

[6] Gutman, I., "Distance of line graphs", Graph Theory Notes New York 31 (1996), 49 - 52.

[7] Gutman, I. and Estrada, E., "Topological indices based on the line graph of the molecular graph", J. Chem. Inf. and Comput. Sci. 36(3) (1996), 541 - 543. 
[8] Gutman, I. and Pavlović, L., "More on distance of line graphs", Graph Theory Notes New York 33 (1997), 14 - 18

[9] Gutman, I., Popović, L., Mishra, B.K., Kuanar, M., Estrada, E. and Guevara, N., "Applications of line graphs in physical chemistry: Predicting the surface tensions of alkanes", $J$. Serb. Chem. Soc. 62(3) (1997), 1025 - 1029.

[10] Gutman, I., Yeh, Y., Lee, S. and Luo, Y., "Some recent results in the theory of the Wiener number", Indian J. Chem. 32A (1993), 651 - 661.

[11] Harary, F., Graph Theory, Narosa Publishing House, New Delhi, 1999.

[12] Merris, R., "An edge version of the matrix - tree theorem and the Wiener index", Lin. Multilin. Algebra 25 (1989), 291 - 296.

[13] Mohar, B., "The Laplacian spectrum of graphs", in: Graph Theory, Combinatorics and Applications, (Eds. Y. Alavi, G. Chartrand, O. R. Ollermann and A. J. Schwenk), Wiley, New York, 1991.

[14] Nikolić, S., Trinajstić, N. and Mihalić, Z., "The Wiener index: development and applications", Croat. Chem. Acta 68 (1995), 105 - 129.

[15] Ramane, H.S., Revankar, D.S., Gutman, I. and Walikar, H.B., "Distance spectra and distance energies of iterated line graphs of regular graphs", Publ. de L'Inst. Math. 85(99) (2009), 39 -46 .

[16] Randić, M., "In search for graph invariants of chemical interest", J. Mol. Struct. 300 (1993), $551-571$.

[17] Walikar, H.B., Shigehalli, V.S. and Ramane, H.S., "Bounds on the Wiener number of a graph", MATCH Comm. Math. Comp. Chem. 50 (2004), 117 - 132.

[18] Wiener, H., "Structural determination of paraffin boiling points", J. Amer. Chem. Soc. 69 (1947), $17-20$. 